\title{
On the final boundary value problems in linear thermoelasticity
}

\author{
Stan Chiriţă
}

Received: 24 January 2012 / Accepted: 3 July 2012 / Published online: 25 July 2012

(C) Springer Science+Business Media B.V. 2012

\begin{abstract}
In the present study we derive some uniqueness criteria for solutions of the Cauchy problem for the standard equations of dynamical linear thermoelasticity backward in time. We use Lagrange-Brun identities combined with some differential inequalities in order to show that the final boundary value problem associated with the linear thermoelasticity backward in time has at most one solution in appropriate classes of displacement-temperature fields. The uniqueness results are obtained under the assumptions that the density mass and the specific heat are strictly positive and the conductivity tensor is positive definite.
\end{abstract}

Keywords Linear thermoelasticity backward in time - Uniqueness results · Lagrange-Brun identity method

\section{Introduction}

The uniqueness and continuous dependence of the thermoelastic processes forward in time have been extensively studied in literature (see, e.g. Brun [1, 2],

\footnotetext{
S. Chiriţă $(\bowtie)$

Department of Mathematics, “Al. I. Cuza” University of Iaşi, Blvd. Carol I, no. 11, 700506 Iaşi, Romania e-mail:schirita@uaic.ro

S. Chiriţă

"Octav Mayer" Mathematics Institute, Romanian Academy of Science, Iaşi Branch, Bd. Carol I, no. 8, 700506 Iaşi, Romania
}

Knops and Payne [3], Levine [4], Wilkes [5], Rionero and Chiriţă [6], Ieşan [7, 8]).

On the other hand, the backward in time problems in thermoelasticity have been considered in many studies (see, for example, Ames and Payne [9], Ciarletta and Chiriţă [10, 11], Ciarletta [12], Iovane and Passarella [13] and Passarella and Tibullo [14]). The question of determining suitable constraint restrictions that will stabilize the problem against errors in the final data was treated firstly by Ames and Payne [9] under a class of restrictive assumptions placed on the thermoelastic coefficients. More contribution in this connection was given by Ciarletta [12], where several uniqueness results for the backward in time thermoelastic problems are established. The main arguments are based on a combination of some Lagrange identities with a Gronwall type inequality and, among other hypotheses, on the assumption of negativeness of the specific heat. We have to outline that Koch and Lasiecka [15] establish backward uniqueness results for thermoelastic plates and thermoelastic waves with time dependent coefficients by means of appropriate Carleman estimates.

As it is well known the final boundary value problems associated to the linear thermoelasticity lead to so-called ill posed problems. In order to stabilize such kind of problems many different techniques have been developed in literature such as those of "solving" ill posed problems for equations of evolution. Some of these involve the altering of the governing equations in such a way as to make such problems well posed. 
Others involve changing the initial and/or boundary conditions again in such a way as to make the problems well posed. This last technique consists in introducing a combination of the initial values with those at a later time. By changing the initial conditions one meet the so-called nonstandard problems and in the recent years such problems are intensively studied in literature. To this end we have to cite the papers concerning the study of the heat equation (see, for example, Ames and Payne [16], Ames, Payne and Schaefer [17, 18], Ames, Payne and Song [19] and Song [20]) and those concerning the generalized heat equation (see, for example, Payne, Schaefer and Song [21]) and those regarding fluid flows (see, e.g. Ames, Payne and Song [19], Payne, Schaefer and Song [22] and Quintanilla and Straughan [23]) as well as those related to linear thermoelasticity (see, e.g. Chiriţă [24] and Chiriţă and Ciarletta [25]). Still others involve constraining solutions to lie in a certain constraint set. It is this latter method which we apply in the present study.

More precisely, here we study the uniqueness for the thermoelastic processes backward in time. We derive uniqueness criteria for solutions of the Cauchy problem for the standard equations of dynamical linear thermoelasticity backward in time. To this end we use the Lagrange-Brun identities combined with some differential inequalities in order to show that the final boundary value problem associated with the linear thermoelasticity backward in time has at most one solution in some appropriate classes of displacementtemperature fields, provided mild hypotheses upon the thermoelastic coefficients are assumed. In fact, we assume that the density mass and the specific heat are strictly positive and the conductivity tensor is positive definite and then we establish uniqueness results in an appropriate class of displacement-temperature fields. This class of displacement-temperature fields is consistently enlarged when the negative semi-definiteness of the elasticity tensor is assumed. The results presented here complete those reported by Ciarletta in [12].

\section{Formulation of problem}

Throughout this paper we assume that the properly regular region $B \subset \mathbb{R}^{3}$ is filled by an inhomogeneous and anisotropic thermoelastic material. We denote by $\partial B$ the boundary surface of $B$. Further, we consider the final boundary value problems associated with the linear theory of thermoelasticity on the time interval $(-\infty, 0]$. The governing equations of the linear theory of anisotropic and inhomogeneous thermoelasticity are given by

- the evolution equations

$S_{j i, j}+b_{i}=\varrho \ddot{u}_{i}$,

- the equation of energy

$-q_{i, i}+h+\theta_{0} M_{i j} \dot{e}_{i j}=c \dot{\theta}$

in $B \times(-\infty, 0)$,

- the constitutive equations

$S_{i j}=C_{i j k l} e_{k l}+M_{i j} \theta$,

$q_{i}=-K_{i j} g_{j}$,

and

- the geometrical relations

$e_{i j}=\frac{1}{2}\left(u_{i, j}+u_{j, i}\right)$,

$g_{i}=\theta_{, i}$

in $\bar{B} \times(-\infty, 0]$.

Here $u_{i}$ are the components of the displacement vector, $\theta$ is the temperature variation from the uniform reference temperature $\theta_{0}, e_{i j}$ are the components of the strain tensor, $g_{i}$ are the components of the thermal gradient vector, $S_{i j}$ are the components of the stress tensor, $q_{i}$ are the components of the heat flux vector, $b_{i}$ represent the components of the external body force vector per unit volume and $h$ is the heat supply per unit volume. Furthermore, $\varrho$ is the mass density, $C_{i j k l}$ are the components of the elasticity tensor, $M_{i j}$ are the components of the stress-temperature tensor, $c$ is the specific heat, and $K_{i j}$ are the components of the conductivity tensor. In what follows we assume that the mass density $\varrho$ and the specific heat $c$ are continuous functions of $\mathbf{x}$ on $B$. Moreover, we assume that the elasticity tensor, the stress-temperature tensor and the conductivity tensor are continuous differentiable functions of $\mathbf{x}$ on $\bar{B}$ and satisfy the symmetry relations

$C_{i j k l}=C_{k l i j}=C_{j i k l}$,

$M_{i j}=M_{j i}, \quad K_{i j}=K_{j i}$.

The subscripts $i, j, k, l$ take values $1,2,3$ and summation is implied by index repetition. Moreover, a superposed dot denotes differentiation with respect to time and a subscript comma indicates partial differentiation. 
Since we are considering the coupled theory of thermoelasticity, it follows that we have

$m=\left(M_{i j} M_{i j}\right)^{1 / 2}>0$.

Further, we set

$m^{*}=\left(M_{i j, k} M_{i j, k}\right)^{1 / 2} \geq 0$.

In this paper we consider the final boundary value problem $(\mathcal{P})$ defined by relations $(2.1)-(2.4)$, the final conditions

$u_{i}(\mathbf{x}, 0)=u_{i}^{0}(\mathbf{x}), \quad \dot{u}_{i}(\mathbf{x}, 0)=\dot{u}_{i}^{0}(\mathbf{x})$,

$\theta(\mathbf{x}, 0)=\theta^{0}(\mathbf{x}), \quad \mathbf{x} \in \bar{B}$,

and the boundary conditions

$$
\begin{aligned}
& u_{i}(\mathbf{x}, t)=\tilde{u}_{i}(\mathbf{x}, t) \quad \text { on } \bar{\Sigma}_{1} \times(-\infty, 0], \\
& s_{i}(\mathbf{x}, t)=\tilde{s}_{i}(\mathbf{x}, t) \quad \text { on } \Sigma_{2} \times(-\infty, 0], \\
& \theta(\mathbf{x}, t)=\tilde{\theta}(\mathbf{x}, t) \quad \text { on } \bar{\Sigma}_{3} \times(-\infty, 0], \\
& q(\mathbf{x}, t)=\tilde{q}(\mathbf{x}, t) \quad \text { on } \Sigma_{4} \times(-\infty, 0],
\end{aligned}
$$

where $u_{i}^{0}, \dot{u}_{i}^{0}, \theta^{0}, \tilde{u}_{i}, \tilde{s}_{i}, \tilde{\theta}$ and $\tilde{q}$ are prescribed functions and

$$
\begin{aligned}
& s_{i}(\mathbf{x}, t)=S_{j i}(\mathbf{x}, t) n_{j}, \\
& q(\mathbf{x}, t)=q_{i}(\mathbf{x}, t) n_{i},
\end{aligned}
$$

$n_{i}$ are the components of the outward unit normal vector to the boundary surface and $\Sigma_{1}, \Sigma_{2}, \Sigma_{3}$ and $\Sigma_{4}$ are subsurfaces of $\partial B$, such that $\bar{\Sigma}_{1} \cup \Sigma_{2}=\bar{\Sigma}_{3} \cup \Sigma_{4}=$ $\partial B$ and $\Sigma_{1} \cap \Sigma_{2}=\Sigma_{3} \cap \Sigma_{4}=\emptyset$.

In the present paper we are interested to establish the uniqueness of solutions of the final boundary value problem $(\mathcal{P})$ under mild conditions upon the thermoelastic coefficients. To this end, as in [12], we transform $(\mathcal{P})$, by making $t \rightarrow-t$ and by using appropriate notations, in the initial boundary value problem $\left(\mathcal{P}^{*}\right)$ defined by the equations

$$
\begin{aligned}
& \left(C_{j i k l} u_{k, l}+M_{j i} \theta\right)_{, j}+b_{i}=\varrho \ddot{u}_{i}, \\
& \left(K_{i j} \theta,{ }_{, j}\right)_{, i}-\theta_{0} M_{i j} \dot{u}_{i, j}+h=-c \dot{\theta},
\end{aligned}
$$

in $B \times(0, \infty)$, with the initial conditions

$$
\begin{aligned}
& u_{i}(\mathbf{x}, 0)=u_{i}^{0}(\mathbf{x}), \quad \dot{u}_{i}(\mathbf{x}, 0)=\dot{u}_{i}^{0}(\mathbf{x}), \\
& \theta(\mathbf{x}, 0)=\theta^{0}(\mathbf{x}), \quad \mathbf{x} \in \bar{B},
\end{aligned}
$$

and the boundary conditions

$$
\begin{aligned}
& u_{i}(\mathbf{x}, t)=\tilde{u}_{i}(\mathbf{x}, t) \quad \text { on } \bar{\Sigma}_{1} \times[0, \infty), \\
& s_{i}(\mathbf{x}, t)=\tilde{s}_{i}(\mathbf{x}, t) \quad \text { on } \Sigma_{2} \times[0, \infty), \\
& \theta(\mathbf{x}, t)=\tilde{\theta}(\mathbf{x}, t) \quad \text { on } \bar{\Sigma}_{3} \times[0, \infty), \\
& q(\mathbf{x}, t)=\tilde{q}(\mathbf{x}, t) \quad \text { on } \Sigma_{4} \times[0, \infty) .
\end{aligned}
$$

In order to establish uniqueness of solutions to the initial boundary value problem $\left(\mathcal{P}^{*}\right)$ it is sufficient to prove that the zero external given data, that is $\mathcal{D}=\left\{b_{i}, h ; u_{i}^{0}, \dot{u}_{i}^{0}, \theta^{0} ; \tilde{u}_{i}, \tilde{s}_{i}, \tilde{\theta}, \tilde{q}\right\}=0$, implies that the corresponding solution $U=\left\{u_{i}, \theta\right\}(\mathbf{x}, t)$ is vanishing on $\bar{B} \times[0, \infty)$. To prove this we note that for any solution $U=\left\{u_{i}, \theta\right\}(\mathbf{x}, t)$ of the initial boundary value problem $\left(\mathcal{P}^{*}\right)$ corresponding to $\mathcal{D}=0$, we have the following identities (see, for example, Ciarletta [12], relations (42) to (46)):

$$
\begin{aligned}
& \int_{B}\left[\varrho \dot{u}_{i}(t) \dot{u}_{i}(t)+C_{i j k l} e_{i j}(t) e_{k l}(t)\right] d v \\
& \quad=-2 \int_{0}^{t} \int_{B} M_{i j} \theta(s) \dot{e}_{i j}(s) d v d s, \\
& \int_{B} \frac{1}{\theta_{0}} c \theta^{2}(t) d v-2 \int_{0}^{t} \int_{B} \frac{1}{\theta_{0}} K_{i j} g_{i}(s) g_{j}(s) d v d s \\
& \quad=2 \int_{0}^{t} \int_{B} M_{i j} \theta(s) \dot{e}_{i j}(s) d v d s,
\end{aligned}
$$

$$
\begin{gathered}
\int_{B}\left[\varrho \dot{u}_{i}(t) \dot{u}_{i}(t)+C_{i j k l} e_{i j}(t) e_{k l}(t)+\frac{1}{\theta_{0}} c \theta^{2}(t)\right] d v \\
-2 \int_{0}^{t} \int_{B} \frac{1}{\theta_{0}} K_{i j} g_{i}(s) g_{j}(s) d v d s=0
\end{gathered}
$$

$$
\begin{aligned}
& \int_{B} \varrho \dot{u}_{i}(t) \dot{u}_{i}(t) d v \\
& \quad=\int_{B}\left[C_{i j k l} e_{i j}(t) e_{k l}(t)+\frac{1}{\theta_{0}} c \theta^{2}(t)\right] d v,
\end{aligned}
$$

$\int_{B} \varrho \dot{u}_{i}(t) \dot{u}_{i}(t) d v=\int_{0}^{t} \int_{B} \frac{1}{\theta_{0}} K_{i j} g_{i}(s) g_{j}(s) d v d s$,

for all $t \in[0, \infty)$.

\section{Uniqueness results}

The uniqueness results established in the present section are based in principal on the assumption that the specific heat is strictly positive in $B$. The case when the specific heat is non-positive in $B$ was studied by Ciarletta [12]. For convenience, we first recall the uniqueness results established by Ciarletta [12].

Theorem 3.1 Suppose that the density mass $\varrho$ is strictly positive on $B$ and $K_{i j}$ is a positive definite tensor and meas $\Sigma_{4}=0$. Moreover, we assume one of the following hypotheses: 
(i) the elasticity tensor is a positive semi-definite tensor;

(ii) $c \leq 0$.

Then the final boundary value problem $\left(\mathcal{P}^{*}\right)$ has at most one solution.

Our main result concerning the uniqueness problem is embedded in the next two theorems.

Theorem 3.2 Suppose that the density mass $\varrho$ is strictly positive on $B, K_{i j}$ is a positive definite tensor, $C_{i j k l}$ is a negative semi-definite tensor and $c>0$ in $B$. Then there exists a strictly positive constant $\alpha$ so that, in the class of displacement-temperature fields $\left\{u_{i}, \theta\right\}(\mathbf{x}, t)$ defined on $\bar{B} \times[0, \infty)$, that satisfy

$\int_{0}^{t} \int_{B} \frac{1}{\theta_{0}} K_{i j} g_{i}(s) g_{j}(s) d v d s \leq M_{1}^{2} e^{\alpha t}$,

for all $t \in[0, \infty)$, with $M_{1}=$ constant, the initial boundary value problem $\left(\mathcal{P}^{*}\right)$ has at most one solution, provided meas $\Sigma_{3} \neq 0$.

Proof Since $K_{i j}$ is a positive definite tensor it follows that

$\kappa_{0} \xi_{i} \xi_{i} \leq K_{i j} \xi_{i} \xi_{j}$,

for all vectors $\left(\xi_{1}, \xi_{2}, \xi_{3}\right)$, with $\kappa_{0}$ a strictly positive constant related to the minimum eigenvalue of the conductivity tensor.

Let us consider $\left\{u_{i}, \theta\right\}(\mathbf{x}, t)$ be a solution of the initial boundary value problem $\left(\mathcal{P}^{*}\right)$ corresponding to zero external given data, that is $\mathcal{D}=\left\{b_{i}, h ; u_{i}^{0}, \dot{u}_{i}^{0}, \theta^{0}\right.$; $\left.\tilde{u}_{i}, \tilde{s}_{i}, \tilde{\theta}, \tilde{q}\right\}=0$. We have to prove that $\left\{u_{i}, \theta\right\}(\mathbf{x}, t)=$ 0 in $\bar{B} \times[0, \infty)$. To this end we note that the identities (2.14)-(2.18) hold true. Then by combining the identities (2.17) and (2.18), we have

$$
\begin{gathered}
\int_{B}\left[C_{i j k l} e_{i j}(t) e_{k l}(t)+\frac{1}{\theta_{0}} c \theta^{2}(t)\right] d v \\
=\int_{0}^{t} \int_{B} \frac{1}{\theta_{0}} K_{i j} g_{i}(s) g_{j}(s) d v d s,
\end{gathered}
$$

for all $t \in[0, \infty)$.

Further, we note that in the case when meas $\Sigma_{3} \neq 0$, we have

$\int_{B} \theta_{, i}(t) \theta_{, i}(t) d v \geq \lambda \int_{B} \theta^{2}(t) d v$,

where $\lambda>0$ is the smallest eigenvalue for the fixed membrane problem for $B$.
Since the elasticity tensor $C_{i j k l}$ is a negative semidefinite tensor, it follows, from the identity (3.3), that

$\int_{0}^{t} \int_{B} \frac{1}{\theta_{0}} K_{i j} g_{i}(s) g_{j}(s) d v d s \leq \int_{B} \frac{1}{\theta_{0}} c \theta^{2}(t) d v$,

for all $t \in[0, \infty)$ and hence, by the use of (3.2) and (3.4), we obtain

$$
\begin{aligned}
& \int_{0}^{t} \int_{B} \frac{1}{\theta_{0}} K_{i j} g_{i}(s) g_{j}(s) d v d s \\
& \quad \leq \omega^{2} \int_{B} \frac{1}{\theta_{0}} K_{i j} g_{i}(t) g_{j}(t) d v,
\end{aligned}
$$

for all $t \in[0, \infty)$, with

$\omega^{2}=\frac{\sup _{\bar{B}} c}{\lambda \kappa_{0}}$.

Further, we set

$\varphi^{2}(t)=\int_{0}^{t} \int_{B} \frac{1}{\theta_{0}} K_{i j} g_{i}(s) g_{j}(s) d v d s$,

for all $t \in[0, \infty)$, and note that (3.6) becomes

$\varphi^{2}(t) \leq 2 \omega^{2} \varphi(t) \dot{\varphi}(t), \quad t \in[0, \infty)$.

Now, it is clear that if $\varphi(t)=0$ for all $t \in[0, \infty)$, then it follows that

$g_{i}(\mathbf{x}, t)=0 \quad$ in $B \times[0, \infty)$

and hence, recalling that meas $\Sigma_{3} \neq 0$, we deduce that

$\theta(\mathbf{x}, t)=0 \quad$ in $\bar{B} \times[0, \infty)$.

Furthermore, by substituting (3.10) into (2.18) and then by using the zero initial conditions, we get

$u_{i}(\mathbf{x}, t)=0 \quad$ in $\bar{B} \times[0, \infty)$,

that is the required uniqueness result.

Let us now suppose that there exists $\tau \in(0, \infty)$ so that $\varphi(\tau)>0$ and hence we have

$\varphi(t)>0$ for all $t \in[\tau, \infty)$.

Then (3.9) gives

$\frac{d}{d t}\left[\varphi(t) e^{-t /\left(2 \omega^{2}\right)}\right] \geq 0 \quad$ for all $t \in[\tau, \infty)$,

and hence, we deduce that

$$
\begin{aligned}
\varphi(\tau) e^{-\tau /\left(2 \omega^{2}\right)} & \leq \varphi(t) e^{-t /\left(2 \omega^{2}\right)} \\
& \leq \lim _{t \rightarrow \infty}\left[\varphi(t) e^{-t /\left(2 \omega^{2}\right)}\right]
\end{aligned}
$$

for all $t \in[\tau, \infty)$. Consequently, if we choose

$0 \leq \alpha<\frac{1}{\omega^{2}}$, 
then the hypothesis expressed by (3.1) implies that

$\lim _{t \rightarrow \infty}\left[\varphi(t) e^{-t /\left(2 \omega^{2}\right)}\right]=0$

and therefore, $(3.15)$ gives

$\varphi(t)=0$ for all $t \in[\tau, \infty)$,

a relation that proves that the relation (3.13) cannot hold true. Thus, we have only the possibility expressed by (3.10) and so we have the uniqueness result and the proof is complete.

Remark 1 It can be seen that this theorem completes the study made by Ciarletta [12]. The novel contribution here is that our uniqueness result is obtained without recourse to the assumption that $c \leq 0$ in $B$. Instead we assume that the solution of the initial boundary value problem $\left(\mathcal{P}^{*}\right)$ belongs to the class of displacement-temperature fields $\left\{u_{i}, \theta\right\}(\mathbf{x}, t)$ that satisfy the constraint (3.1).

Remark 2 The negative semi-definiteness condition on the elasticity tensor $C_{i j k l}$ can be removed from the above theorem. Instead we have to restrict the constraint set defined by (3.1). The corresponding uniqueness result is embedded in the following theorem.

Theorem 3.3 Suppose that the density mass $\varrho$ and the specific heat $c$ are strictly positive on $B$ and $K_{i j}$ is a positive definite tensor and meas $\Sigma_{4}=0$. Then, in the class of displacement-temperature fields $\left\{u_{i}, \theta\right\}(\mathbf{x}, t)$ defined on $\bar{B} \times[0, \infty)$, that satisfy

$\lim _{t \rightarrow \infty} \int_{0}^{t} \int_{0}^{s} \int_{B} \frac{1}{\theta_{0}} K_{i j} g_{i}(\tau) g_{j}(\tau) d v d \tau d s=0$,

the initial boundary value problem $\left(\mathcal{P}^{*}\right)$ has at most one solution.

Proof Let $\left\{u_{i}, \theta\right\}(\mathbf{x}, t)$ be a solution of the initial boundary value problem $\left(\mathcal{P}^{*}\right)$ corresponding to zero external given data, that is $\mathcal{D}=\left\{b_{i}, h ; u_{i}^{0}, \dot{u}_{i}^{0}, \theta^{0} ; \tilde{u}_{i}\right.$, $\left.\tilde{s}_{i}, \tilde{\theta}, \tilde{q}\right\}=0$. Following Ciarletta [12], by means of the Cauchy-Schwarz inequality and the arithmeticgeometric mean inequality, we obtain the following estimate

$$
\begin{aligned}
& -2 \int_{0}^{t} \int_{B} M_{i j} \theta(s) \dot{e}_{i j}(s) d v d s \\
& \leq \frac{M_{2}}{\varepsilon \sqrt{\kappa_{0}}} \int_{0}^{t} \int_{B} \varrho \dot{u}_{i}(s) \dot{u}_{i}(s) d v d s \\
& \quad+\frac{4 \theta_{0} M_{2}}{\sqrt{\kappa_{0}}} \varepsilon \int_{0}^{t} \int_{B} \frac{1}{\theta_{0}} K_{i j} g_{i}(s) g_{j}(s) d v d s,
\end{aligned}
$$

for all $t \in[0, \infty)$, with the constant $M_{2}$ defined by

$$
M_{2}=\max \left\{\sup _{\bar{B}} \frac{m}{\sqrt{\varrho}}, \sup _{\bar{B}} \frac{m^{*}}{\sqrt{\lambda \varrho}}\right\}
$$

and $\varepsilon$ is a positive constant which arises through use of the arithmetic-geometric mean inequality and may be selected judiciously. The quantity $\lambda$ is the smallest eigenvalue for the fixed membrane problem for $B$.

Using (2.18) and (3.20) in (2.15), we are led to the inequality

$$
\begin{aligned}
(2- & \left.\frac{4 \theta_{0} M_{2}}{\sqrt{\kappa_{0}}} \varepsilon\right) \int_{B} \varrho \dot{u}_{i}(t) \dot{u}_{i}(t) d v \\
\leq & \int_{B} \frac{1}{\theta_{0}} c \theta^{2}(t) d v \\
& +\frac{M_{2}}{\varepsilon \sqrt{\kappa_{0}}} \int_{0}^{t} \int_{B} \varrho \dot{u}_{i}(s) \dot{u}_{i}(s) d v d s,
\end{aligned}
$$

for all $t \in[0, \infty)$. An appropriate choice for $\varepsilon$ in inequality (3.22) is

$\varepsilon=\frac{\sqrt{\kappa_{0}}}{4 \theta_{0} M_{2}}$

and then we have the following inequality

$\psi(t) \leq \alpha(t)+M_{0} \int_{0}^{t} \psi(s) d s, \quad t \in[0, \infty)$,

where

$\psi(t)=\int_{B} \varrho \dot{u}_{i}(t) \dot{u}_{i}(t) d v$,

$\alpha(t)=\int_{B} \frac{1}{\theta_{0}} c \theta^{2}(t) d v$,

$M_{0}=\frac{4 \theta_{0} M_{2}^{2}}{\kappa_{0}}$.

Further, we set

$\chi(t)=e^{-M_{0} t} M_{0} \int_{0}^{t} \psi(s) d s$

and note that

$$
\begin{aligned}
\dot{\chi}(t) & =M_{0} e^{-M_{0} t}\left[\psi(t)-M_{0} \int_{0}^{t} \psi(s) d s\right] \\
& \leq M_{0} e^{-M_{0} t} \alpha(t)
\end{aligned}
$$

and therefore, we have

$\chi(t) \leq M_{0} \int_{0}^{t} e^{-M_{0} s} \alpha(s) d s, \quad t \in[0, \infty)$.

Concluding, from (3.28) and (3.30) we obtain

$\int_{0}^{t} \psi(s) d s \leq \int_{0}^{t} e^{-M_{0}(s-t)} \alpha(s) d s$,

for all $t \in[0, \infty)$. 
On the other hand, by using (3.2), (3.4) and (3.26), we have

$\alpha(t) \leq \frac{\sup _{\bar{B}} c}{\lambda \kappa_{0}} \int_{B} \frac{1}{\theta_{0}} K_{i j} g_{i}(t) g_{j}(t) d v$,

which, when used in (3.31), furnishes

$\int_{0}^{t} \psi(s) d s \leq \frac{\sup _{\bar{B}} c}{\lambda \kappa_{0}} e^{M_{0} t} \psi(t)$,

for all $t \in[0, \infty)$.

Furthermore, we set

$\phi(t)=\left(\int_{0}^{t} \psi(s) d s\right)^{1 / 2}, \quad t \in[0, \infty)$,

so that (3.33) becomes

$\phi^{2}(t) \leq \frac{2 \sup _{\bar{B}} c}{\lambda \kappa_{0}} e^{M_{0} t} \phi(t) \dot{\phi}(t), \quad t \in[0, \infty)$.

Let us first consider the case when

$\phi(t)=\left(\int_{0}^{t} \psi(s) d s\right)^{1 / 2}=0, \quad t \in[0, \infty)$.

Then it follows from (3.25) and the zero initial data that

$u_{i}(\mathbf{x}, t)=0 \quad$ in $\bar{B} \times[0, \infty)$,

and, moreover, by means of the identity (2.18) and the fact that $c>0$ in $\bar{B}$, we have

$\theta(\mathbf{x}, t)=0 \quad$ in $\bar{B} \times[0, \infty)$,

that is we are led to the required uniqueness result.

Let us now consider that there exists $\tau \in(0, \infty)$ so that $\phi(\tau)>0$ and hence we have

$\phi(t)>0$ for all $t \in[\tau, \infty)$.

Then (3.35) implies

$\frac{d}{d t}\left[e^{\xi(t)} \phi(t)\right] \geq 0 \quad$ for all $t \in[\tau, \infty)$,

with

$\xi(t)=\frac{\lambda \kappa_{0}}{2 M_{0} \sup _{\bar{B}} c}\left(e^{-M_{0} t}-1\right)$.

Thus, from (3.40) we deduce

$e^{\xi(\tau)} \phi(\tau) \leq e^{\xi(t)} \phi(t) \leq \lim _{t \rightarrow \infty}\left[e^{\xi(t)} \phi(t)\right]$,

for all $t \in[\tau, \infty)$. In view of the hypothesis (3.19) and by using (3.25), we obtain

$$
\begin{aligned}
\lim _{t \rightarrow \infty}\left[e^{\xi(t)} \phi(t)\right]= & e^{-\frac{\lambda \kappa_{0}}{2 M_{0} \sup \bar{B}}[}\left[\lim _{t \rightarrow \infty} \int_{0}^{t} \int_{0}^{s} \int_{B} \frac{1}{\theta_{0}} K_{i j}\right. \\
& \left.\times g_{i}(\tau) g_{j}(\tau) d v d \tau d s\right]^{1 / 2} \\
= & 0
\end{aligned}
$$

and therefore, (3.42) implies that

$\phi(t)=0 \quad$ for all $t \in[\tau, \infty)$,

a relation that is in contradiction with our hypothesis expressed in (3.39).

Concluding, we have only the possibility expressed by (3.36), that is we have (3.37) and (3.38) and hence the proof is complete.

Remark 3 It can be easily seen that the assumption embedded in (3.19) is more restrictive than that given in (3.1) and so the class of displacement-temperature fields $\left\{u_{i}, \theta\right\}(\mathbf{x}, t)$, where the uniqueness result holds true, is essentially reduced.

\section{References}

1. Brun L (1965) Sur l'unicité en thermoelasticité dynamique et diverses expressions analogues à la formule de Clapeyron. C R Acad Sci Paris 261:2584-2587

2. Brun L (1969) Méthodes énergétiques dans les systèmes évolutifs linéaires, Première partie : Separation des énergies, Deuxième partie : Théorèmes d'unicité. J Méc 8:125-166. 167-192

3. Knops RJ, Payne LE (1970) On uniqueness and continuous data dependence in dynamical problems of linear thermoelasticity. Int J Solids Struct 6:1173-1184

4. Levine HA (1970) On a theorem of Knops and Payne in dynamical thermoelasticity. Arch Ration Mech Anal 38:290-307

5. Wilkes NS (1980) Continuous dependence and instability in linear thermoelasticity. SIAM J Appl Math 11:292-299

6. Rionero S, Chiriţă S (1987) The Lagrange identity method in linear thermoelasticity. Int J Eng Sci 25:935-947

7. Ieşan D (1989) On some theorems in thermoelastodynamics. Rev Roum Sci Tech, Sér Méc Appl 34:101-111

8. Ieşan D (1989) Reciprocity, uniqueness and minimum principles in the dynamic theory of thermoelasticity. J Therm Stresses 12:465-482

9. Ames KA, Payne LE (1991) Stabilizing solutions of the equations of dynamical linear thermoelasticity backward in time. Stab Appl Anal Contin Media 1:243-260

10. Ciarletta M, Chiriţă S (2001) Spatial behavior in linear thermoelasticity backward in time. In: Chao CK, Lin CY (eds) Proceedings of the fourth international congress on thermal stresses, Osaka, Japan, pp 485-488

11. Ciarletta M, Chiriţă S (2002) Asymptotic partition in the linear thermoelasticity backward in time. In: Mathematical models and methods for smart materials, Cortona, 2001. Series on advances in mathematics for applied sciences, vol 62. World Scientific, River Edge, pp 31-41

12. Ciarletta M (2002) On the uniqueness and continuous dependence of solutions in dynamical thermoelasticity backward in time. J Therm Stresses 25:969-984

13. Iovane G, Passarella F (2004) Spatial behaviour in dynamical thermoelasticity backward in time for porous media. J Therm Stresses 27:97-109 
14. Passarella F, Tibullo V (2010) Some results in linear theory of thermoelasticity backward in time for microstretch materials. J Therm Stresses 33:559-576

15. Koch H, Lasiecka I (2007) Backward uniqueness in linear thermoelasticity with time and space variable coefficients. In: Functional analysis and evolution equations: Gunter Lumer volume. Birkhäuser, Basel, pp 389-403

16. Ames KA, Payne LE (1998) Asymptotic behavior for two regularizations of the Cauchy problem for the backward heat equation. Math Models Methods Appl Sci 8:187-202

17. Ames KA, Payne LE, Schaefer PW (2004) On a nonstandard problem for heat conduction in a cylinder. Appl Anal 83:125-133

18. Ames KA, Payne LE, Schaefer PW (2004) Energy and pointwise bounds in some nonstandard parabolic problems. Proc R Soc Edinb A 134:1-9

19. Ames KA, Payne LE, Song JC (2005) On two classes of nonstandard parabolic problems. J Math Anal Appl 311:254-267
20. Song JC (2001) Spatial decay for solutions of Cauchy problems for perturbed heat equations. Math Models Methods Appl Sci 11:797-808

21. Payne LE, Schaefer PW, Song JC (2004) Improved bounds for some nonstandard problems in generalized heat conduction. J Math Anal Appl 298:325-340

22. Payne LE, Schaefer PW, Song JC (2004) Some nonstandard problems in viscous flow. Math Methods Appl Sci 27:2045-2053

23. Quintanilla R, Straughan B (2005) Bounds for some nonstandard problems in porous viscous flow and viscous Green-Naghdi fluids. Proc R Soc Lond A 461:3159-3168

24. Chiriţă S (2009) On some non-standard problems in linear thermoelasticity. J Therm Stresses 32:1256-1269

25. Chiriţă S, Ciarletta M (2010) Spatial behavior for some non-standard problems in linear thermoelasticity without energy dissipation. J Math Anal Appl 367:58-68 